\begin{tabular}{cc}
\hline International Journal of Engineering \& Technology, $7(2.7)(2018) 466-469$ \\
SPC & Website: $w$ ww.sciencepubco.com/index.php/IJET \\
Research Paper & International Journal of Engineering \& Technology \\
\hline
\end{tabular}

\title{
Satellite Image Resolution Enhancement based on Dual-Domain Filtering
}

\author{
K.Rasool Reddy $^{1 *}$, Dr.K.NagaPrakash ${ }^{2}$, Dr.K.Prasanthi Jasmime ${ }^{3}$, M.Tulasidas ${ }^{4}$ \\ ${ }^{1}$ Assistant Professor, Gudlavalleru Engineering College, Gudlavalleru, Andhra Pradesh, India \\ ${ }^{2}$ Professor, Gudlavalleru Engineering College, Gudlavalleru, Andhra Pradesh, India \\ ${ }^{3}$ Professor, ALIET, Vijayawada, Andhra Pradesh, India \\ ${ }^{4}$ Assistant Professor, DHAN, Vijayawada, Andhra Pradesh, India \\ *Corresponding author E-mail: rasoolkamireddy3738@gmail.com
}

\begin{abstract}
Satellite images place vital role in agriculture, Disaster mitigation and geosciences applications. Satellite images include both spatial and temporal resolution, in that spatial resolution is influence the accuracy of ground objects. The main idea of this work is to enhance the resolution of satellite images. In this work, a dual domain filtering based approach is introduced for resolution enhancement (RE). Initially, the source image is subdivided into approximation and detail coefficients by Stationary Wavelet transform (SWT). The detail coefficients are interpolated based on bi-linear interpolation. The interpolated detail coefficients are applied to Non-Local Means (NLM) filter to minimize the artifacts produced by SWT. The filtered detail and approximation coefficients of source image are fed to ISWT to attain high resolution (HR) image. The proposed system is superior to other existing strategies like DWT-NLM and DWT-SWT.
\end{abstract}

Keywords: DWT, Interpolation, NLM Filter, Resolution and SWT

\section{Introduction}

Resolution enhancement is one of key concept in the field of computer-vision applications such as feature extraction, medical imagining, image fusion and image registration etc. Interpolation [1] is one of most popular method for resolution enhancement. Widely used interpolation methods are nearest neighbor, bi-linear, bi-cubic, and Lanczos.

In present decades, several wavelet based image resolution strategies are introduced with conventional techniques (interpolation) namely, DWT [2], SWT [3], DT-CWT [4], Wavelet Zero Padding and cycle-spinning [5], wavelet with directional cycle spinning [6] and DWT based super resolution [7].

In this paper, a dual domain filtering based satellite resolution enhancement approach is introduced using SWT, bi-linear interpolation and NLM. Moreover, SWT is reduces the loss of information compared to DWT. NLMF [8] is used to improve the performance of SWT by minimizing the artifacts. The simulation results of our method are compared to other existing strategies such as DWT-NLM and DWT-SWT [3] in terms of objective quality assessments.

The rest of the work organized as section 2 discusses the preliminaries of the proposed work; section 3 discuss about the proposed method; section 4 discuss about the quality metrics; section 5 discuss about the simulation results and section 5 discuss about the conclusion and future scope.

\section{Preliminaries}

\subsection{Stationary Wavelet Transform (SWT)}

The DWT is not time-invariant transform. To overcome the drawbacks of DWT a new transform is introduced that is Stationary wavelet transform and is also known as Undecimated Wavelet Transform (UWT). The SWT plays very important role in change detection, denoising and pattern recognition etc. In SWT apply LPF and HPF on image data at each level and modify the filters at each stage by zero padding. The below figure 1 represents the 3-level SWT Filter bank.

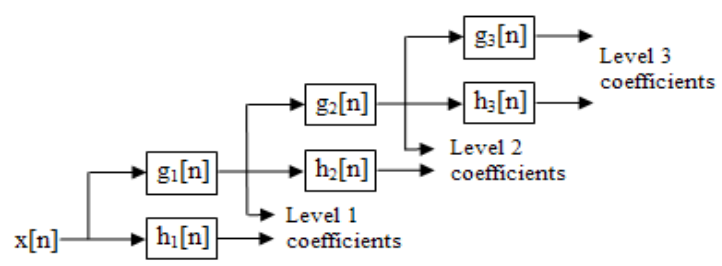

Fig.1: 3-Level SWT Filter Bank

In the below figure 1, filters in each level are interpolated by a factor of 2 and is shown in below figure 2 .

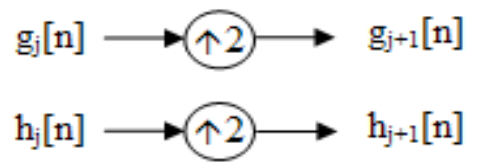

Fig.2: Interpolated version of SWT Filters 


\subsection{Bi-linear Interpolation}

Interpolation is the process to increase the number of pixels in image. There are several interpolations methods are present in that bi-linear interpolation is one. In this method consider the values of 4 closest pixels from the specified co-ordinates and calculate the weighted average based on brightness of the closest pixels and assign that value to the pixel in the output image.

The kernel which is used in interpolation given by

$p(k)=\left\{\begin{array}{r}0,|k|>1 \\ 1-|k|,|k|<1\end{array}\right.$

Where ' $\mathrm{k}$ ' is the distance between points.

\subsection{Non-Local Means Filtering (NLMF)}

Author's [10] introduces a new filter to free from the noise and is known as Non-Local means filtering. In bilateral filter consider only neighborhood pixels since it is a "local filter" but where as in non-local means consider the all pixels in the image. Due that reason non-local means gives superior performance than that of local-means algorithms.

The NLMF is defined as

$\operatorname{NLM}(X)(s)=\sum_{t=X} k(s, t) X(t)$

Here $\mathrm{X}$ represents degraded image and weight $\mathrm{k}(\mathrm{s}, \mathrm{t})$ satisfy the below conditions

$$
0 \leq \mathrm{k}(\mathrm{s}, \mathrm{t}) \leq 1 \text { and } \sum_{t} k(s, t)=1 .
$$

The weights of NLMS algorithm is given by

$k(s, t)=\frac{1}{Z(s)} e^{-\frac{\|v(s)-v(t)\|_{2}^{2}}{h^{2}}}$

Where $Z(s)=\sum_{s} e^{-\frac{\|v(s)-v(t)\|_{2}^{2}}{h^{2}}}$

Here ' $h$ ' used as a controlling parameter of filtering.

\section{Proposed Method}

The below figure 3 represents the satellite image resolution enhancement based on SWT-NLM. In the proposed method initially, 1-level SWT has been employed on the source image to subdivide into approximations (LL) and Detail (LH, HL and $\mathrm{HH})$ coefficients. The detail coefficients are interpolated by a factor of 2 using bi-linear technique. However, due to shiftinvariant property of SWT after interpolation it may produce artifacts. To overcome these problems all interpolated subbands are filtered by NLM. Then, finally perform Inverse SWT on low-frequency and filtered interpolated subbands to obtain resolution enhanced image (or high resolution image).

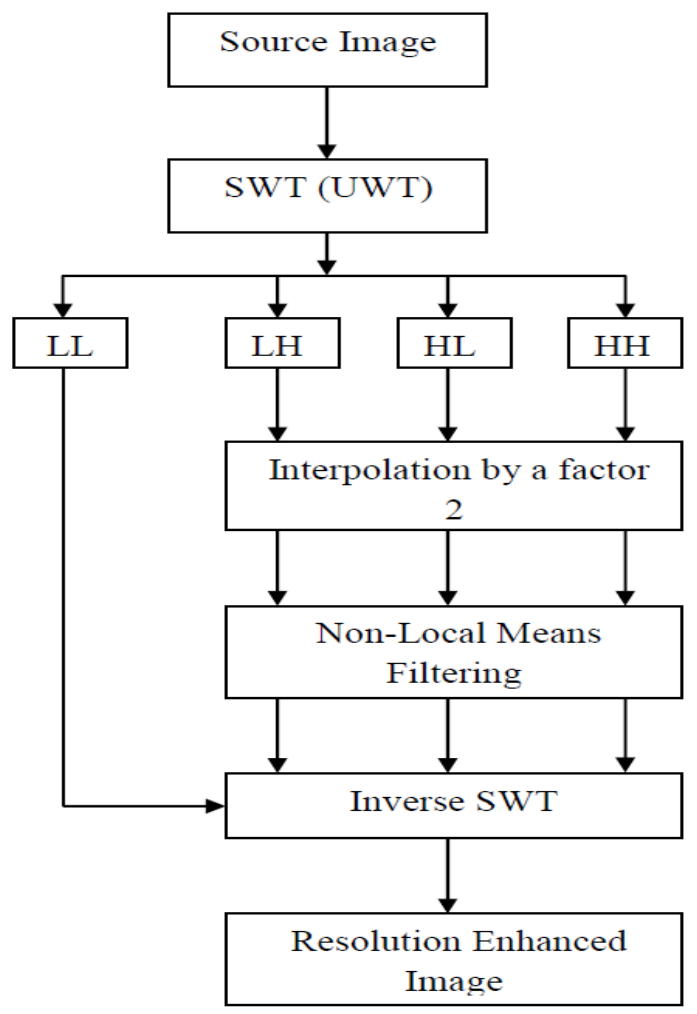

Fig.3: Satellite Resolution Enhancement Based on SWT-NLM

\section{Performance Metrics}

In this section describe various objective image quality metrics to assess the quality of different satellite resolution enhancement strategies. Generally there are three kinds of objective image quality metrics are there in that, only consider Full Reference (FR) quality metrics to assess the quality of an image. The below table 1 represents the list of quality metrics that are used in this work.

Table 1: Quality Metrics

\begin{tabular}{|l|l|l|l|l|}
\hline S. No & Acronym & Abbreviation & Ref. & Description \\
\hline 1 & PSNR & Peak Signal-to-Noise Ratio & {$[9]$} & \multicolumn{1}{|c|}{ PSNR $=10 \log _{10}\left(255^{2} /\right.$ MSE $)$} \\
& & & MSE $=\frac{1}{\mathrm{MN}} \sum_{\mathrm{i}=1}^{\mathrm{M}} \sum_{\mathrm{j}=1}^{\mathrm{N}}\left(a_{i, j}-b_{i, j}\right)^{2}$ \\
\hline 2 & SSIM & Structural Similarity Index Measurement & {$[10]$} & See [10] and practical realization in [14] \\
\hline 3 & VIF & Visual Information Fidelity & {$[11]$} & See [11] and practical realization in [14] \\
\hline 4 & FSIM & Feature Similarity Index Metric & {$[12]$} & See [12] and practical realization in [14] \\
\hline 5 & RFSIM & $\begin{array}{l}\text { Riesz-transform Based Feature Similarity } \\
\text { Metric }\end{array}$ & {$[13]$} & See [13] and practical realization in [14] \\
\hline
\end{tabular}




\section{Results and Discussions}

The below figure 4 shows the enhancement images of various techniques such as DWT-SWT, DWT-NLM and DWT-SWT

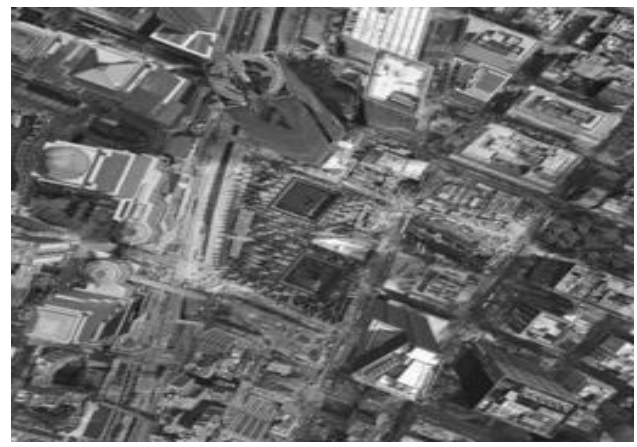

(i)

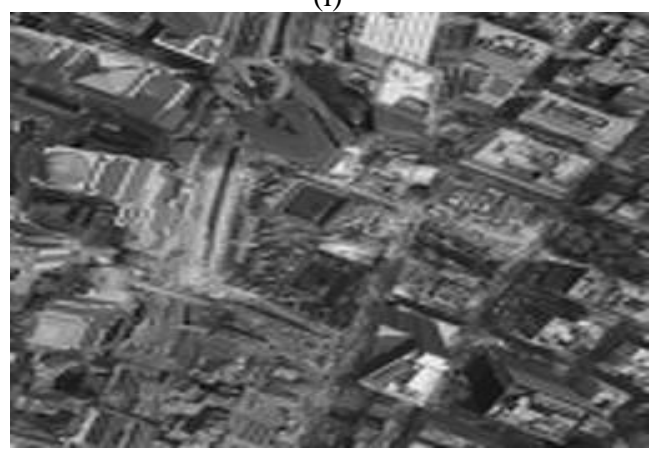

(ii)

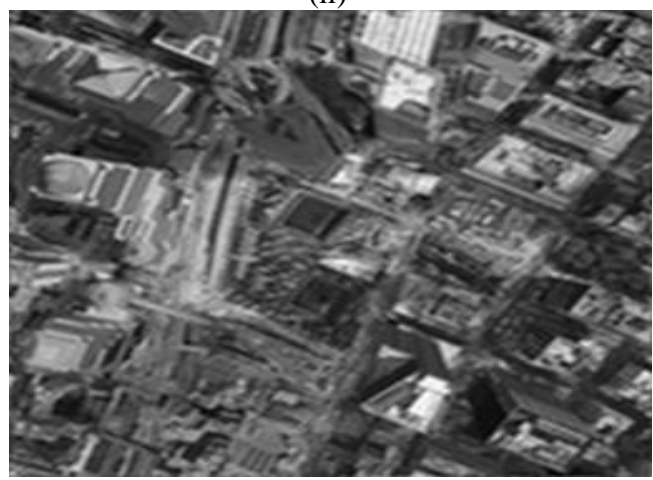

(iii)

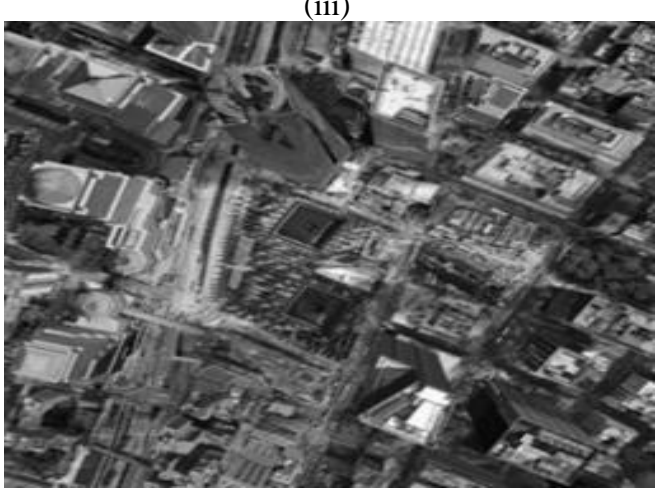

(iv)

Fig. 4: (i) Low resolution "Google earth.jpg" (ii) DWT-SWT (iii) DWT-NLM (iv) Proposed SWT-NLM
As per results mentioned in the table 2 and from figure 4, it clearly said that, the performance of proposed method SWT-NLM is superior to other existing algorithms such as DWT-SWT and DWT-NLM in terms of both visuality and structurality.

Table 2: Quality Metrics for 256×256 Resolution Image

\begin{tabular}{|c|c|c|c|c|c|}
\hline \multirow{3}{*}{ Method } & \multicolumn{5}{|c|}{ Monsoon.jpg } \\
\hline & \multicolumn{5}{|c|}{ Quality Metrics } \\
\hline & PSNR & SSIM & VIF & RFSIM & FSIM \\
\hline $\begin{array}{l}\text { DWT- } \\
\text { SWT }\end{array}$ & 34.6880 & 0.3822 & 1.3425 & 0.9665 & 0.9793 \\
\hline $\begin{array}{l}\text { DWT- } \\
\text { NLM }\end{array}$ & 48.7760 & 0.8429 & 1.3698 & 0.9945 & 0.9983 \\
\hline $\begin{array}{l}\text { SWT- } \\
\text { NLM } \\
\end{array}$ & 50.1185 & 0.8658 & 2.1222 & 0.9959 & 0.9987 \\
\hline \multirow{3}{*}{ Method } & \multicolumn{5}{|c|}{ Google Earth.jpg } \\
\hline & \multicolumn{5}{|c|}{ Quality Metrics } \\
\hline & PSNR & SSIM & VIF & RFSIM & FSIM \\
\hline $\begin{array}{l}\text { DWT- } \\
\text { SWT }\end{array}$ & 36.5505 & 0.2938 & 1.0252 & 0.9727 & 0.9737 \\
\hline $\begin{array}{l}\text { DWT- } \\
\text { NLM }\end{array}$ & 48.1491 & 0.8377 & 1.1384 & 0.9958 & 0.9980 \\
\hline $\begin{array}{l}\text { SWT- } \\
\text { NLM } \\
\end{array}$ & 48.2496 & 0.8658 & 1.9670 & 0.9968 & 0.9987 \\
\hline \multirow{3}{*}{ Method } & \\
\hline & \multicolumn{2}{|c|}{ Quality Metrics } & & & \\
\hline & PSNR & SSIM & VIF & RFSIM & FSIM \\
\hline $\begin{array}{l}\text { DWT- } \\
\text { SWT }\end{array}$ & 35.0988 & 0.3478 & 0.9365 & 0.9640 & 0.9757 \\
\hline $\begin{array}{l}\text { DWT- } \\
\text { NLM }\end{array}$ & 39.6027 & 0.7752 & 1.2610 & 0.9936 & 0.9981 \\
\hline $\begin{array}{l}\text { SWT- } \\
\text { NLM } \\
\end{array}$ & 40.0646 & 0.7930 & 2.1961 & 0.9954 & 0.9988 \\
\hline \multirow{3}{*}{ Method } & \multicolumn{5}{|c|}{ Real-Estate.jpg } \\
\hline & \multicolumn{5}{|c|}{ Quality Metrics } \\
\hline & PSNR & SSIM & VIF & RFSIM & FSIM \\
\hline $\begin{array}{l}\text { DWT- } \\
\text { SWT }\end{array}$ & 38.0830 & 0.4781 & 1.0126 & 0.9760 & 0.9773 \\
\hline $\begin{array}{l}\text { DWT- } \\
\text { NLM }\end{array}$ & 53.2029 & 0.9193 & 1.2720 & 0.9975 & 0.9988 \\
\hline $\begin{array}{l}\text { SWT- } \\
\text { NLM } \\
\end{array}$ & 52.8050 & 0.9367 & 2.7695 & 0.9987 & 0.9992 \\
\hline \multirow{3}{*}{ Method } & \multicolumn{5}{|c|}{ Suomo-Funso.jpg } \\
\hline & \multicolumn{5}{|c|}{ Quality Metrics } \\
\hline & PSNR & SSIM & VIF & RFSIM & FSIM \\
\hline $\begin{array}{l}\text { DWT- } \\
\text { SWT }\end{array}$ & 34.9244 & 0.4533 & 0.9966 & 0.9762 & 0.9813 \\
\hline $\begin{array}{l}\text { DWT- } \\
\text { NLM }\end{array}$ & 52.5363 & 0.9712 & 1.0713 & 0.9993 & 0.9996 \\
\hline $\begin{array}{l}\text { SWT- } \\
\text { NLM }\end{array}$ & 55.6430 & 0.9770 & 2.7480 & 0.9995 & 0.9997 \\
\hline
\end{tabular}




\section{Conclusion}

In this paper a new RE approach based on SWT and NLMF was proposed. First, apply SWT on source image and then interpolate the detail coefficients obtained by DWT, after that remove the distortion introduced by SWT by using NLM Filter. From the simulation results, the proposed SWT-NLM provides superior performance compared to other state-art-of techniques such as DWT-SWT and DWTNLM in terms of visuality and structurality.

\section{References}

[1] Yinji Piao, ll-hong Shin, Hyun Wook Park, "Image Resolution Enhancement using Inter-Subband Correlation in Wavelet Domain", ICIP 2007, pp. I-445I-448.

[2] P. Karunakar,V. Praveen and O. Ravi Kumar "DWTbased satellite image resolution enhancement," Advance in Electronic and Electric Engineering. ISSN 2231-1297, Volume 3, Number 4 (2013), pp. 405-412

[3] Hasan Demirel and Gholamreza Anbarjafar "IMAGE Resolution Enhancement by Using Discrete and Stationary Wavelet Decomposition," IEEE Transactions on Image Processing, Vol. 20, No. 5, May 2011

[4] Hasan Demirel and Gholamreza Anbarjafari, "Satellite Image Resolution Enhancement Using Complex Wavelet Transform" IEEE Geoscience and Remote Sensing Letters, Vol. 7, No. 1, January 2010

[5] Alptekin Temizel and Theo Vlachos, "Wavelet domain image resolution enhancement using cycle-spinning", Electronics Letters, vol. 41, no. 3, pp. 119-121, February 2005.

[6] Alptekin Temizel and Theo Vlachos, "Image resolution upscaling in the wavelet domain using directional cycle spinning," Vol. 14(4), pp: 040501-1, Oct-Dec 2005

[7] Gholamreza Anbarjafari and Hasan Demirel,“ Image Super Resolution Based on Interpolation of Wavelet Domain High Frequency Subbands and the Spatial Domain Input Image"," ETRI Journal Volume 32, Issue 3, pp.390-394, June 2010.

[8] O. Srinu, N.V. Apparao, and K. Rasool Reddy, "A New Approach of Modified ROI-Based Lossy and Lossless Image Compression for Telemedicine Applications", CiiT Digital image processing, Vol. 8, No 8. Aug. 2016

[9] Zhou Wang, Alan C. Bovik, Hamid R. Sheikh, and Eero P. Simoncelli "Image Quality Assessment: From Error Visibility to Structural Similarity", IEEE Transactions on Image Processing, Vol. 13, No. 4, April 2004

[10] Hamid R. Sheikh and Alan C. Bovik, "Image Information And Visual Quality," ICASSP, pp. III-709III-712, May 2004

[11] Kamireddy R.R., Punem S., Jangala S., Chamarthi G.R.D., Srinivas K.Y. "Objective Quality Assessments of Restoration Images" Proceedings of International Conference on Communication and Networks. Advances in Intelligent Systems and Computing, vol 508. pp 255268, Feb 2016.

[12] Lin Zhanga, Lei Zhanga , and Xuanqin Mou, "RFSIM: A Feature Based Image Quality Assessment Metric Using Riesz Transforms" IEEE International Conference on Image Processing, pp. 321-324, September 2010

[13] http://sse.tongji.edu.cn/linzhang/IQA/IQA.html 